\title{
Distribution Power Loss Reduction of Standalone DC Microgrids Using Adaptive Differential Evolution- Based Control for Distributed Battery Systems
}

\author{
Junli Deng ${ }^{1}$, Yuan Mao ${ }^{2}$ and Yun Yang ${ }^{3, *}$ \\ 1 College of Informatics, Huazhong Agricultural University, Wuhan 430000, China; \\ rainbow99@mail.hzau.edu.cn \\ 2 Department of Electrical Engineering, The Hong Kong Polytechnic University, Hong Kong, China; \\ yuan.my.mao@connect.polyu.hk \\ 3 Department of Electrical and Electronic Engineering, The University of Hong Kong, Hong Kong, China \\ * Correspondence: cacalotoyangyun@gmail.com; Tel.: +852-28578419
}

Received: 25 March 2020; Accepted: 22 April 2020; Published: 27 April 2020

\begin{abstract}
With high penetrations of renewable energy sources (RES), distributed battery systems (DBS) are widely adopted in standalone DC microgrids to stabilize the bus voltages by balancing the active power. This paper presents an Adaptive Differential Evolution (ADE)-based hierarchical control for DBS to achieve online distribution power loss mitigation as well as bus voltage regulations in standalone DC microgrids. The hierarchical control comprises two layers, i.e., ADE for the secondary layer and local proportional-integral (PI) control for the primary layer. The secondary layer control provides the bus voltage references for the primary control by optimizing the fitness function, which contains the parameters of the bus voltage deviations and the power loss on the distribution lines. Simultaneously, the state-of-charge $(\mathrm{SoC})$ of the battery packs are controlled by local controllers to prevent over-charge and deep-discharge. Case studies using a Real-Time Digital Simulator (RTDS) validate that the proposed ADE-based hierarchical control can effectively reduce the distribution power loss and regulate the bus voltages within the tolerances in DC microgrids.
\end{abstract}

Keywords: distributed battery system (DBS); DC microgrid; Adaptive Differential Evolution (ADE); hierarchical control; distribution power loss; bus voltage regulation

\section{Introduction}

Due to higher efficiency and easier interconnections for renewable energy sources (RES), DC microgrids have been gaining more attention than their AC counterparts in recent years [1-3]. DC microgrids can be either grid-connected or standalone [4]. For the emerging applications of DC microgrids in data centers, electric ships and smart buildings, etc., standalone DC microgrids seem to be more popular and encourage intensified research activities [5-7]. Typical standalone DC microgrids comprise distributed RES, energy storage systems (ESS), and various types of loads and power electronics interfaces [1-9]. Distributed RES, such as wind energy conversion systems (WECS) and photovoltaic (PV) systems, are the major power supplies of standalone DC microgrids. Various types of loads, such as constant impedance loads, constant power loads and constant current loads, are the main power consumptions of standalone DC microgrids. Due to the intermittent nature of RES, power imbalance between the power supplies and consumption may frequently occur in standalone DC microgrids, which result in undesirable bus voltage fluctuations. To tame the fluctuations, ESS, such as batteries, supercapacitors and flywheels, etc., must be introduced to compensate for the power gap. Generally, power electronics interfaces are required for most RES, ESS, and some types of loads to meet the rated bus voltages. 
Owing to the high-power density and relatively mature technique, batteries are the most widely used ESS in standalone DC microgrids nowadays [10]. As the batteries are usually installed in a distributed manner over the standalone DC microgrids, they are also known as distributed battery systems (DBS). So far, various control strategies have been investigated to control DBS in standalone DC microgrids. Most of these control strategies are droop control and its variants to achieve accurate power/current sharing, state-of-charge (SoC) balance, efficiency optimization of power conversions and economic dispatch etc., [11-19]. In [11], a conventional droop control is adopted to generate output voltage references for local controllers to restore the DC bus voltages based on the output voltages and currents of DBS. However, the conventional droop control may lead to current sharing imbalance when the parameter deviates. Therefore, several adaptive droop control schemes are proposed to eliminate the inequal current sharing of DBS in [12-15]. Besides, the SoC of DBS is balanced by a double-quadrant droop control in order to prolong the lifetime and avoid overuse of batteries in [16]. A multiagent supervisory control is designed to precisely manage the power flow of various isolated DC microgrids in [17]. A multi-objective algorithm is proposed to minimize the cost and lifecycle emissions of standalone PV-wind-diesel systems with battery storage systems in [18]. Economic dispatch of the components in DC microgrids are achieved by minimizing the total operating cost of DBS in [19]. However, mitigating power loss on the distribution lines of standalone DC microgrids have not yet been fully investigated. Similar investigations on DC electric springs in DC microgrids, AC electric springs and DBS in AC microgrids are conducted in [20-22]. However, control of DBS to reduce the distribution power loss reduction of standalone DC microgrids have not been studied.

To bridge the research gap, an adaptive different evolution (ADE)-based centralized control is proposed in this paper to reduce the power loss on distribution lines of standalone DC microgrids online. ADE is a stochastic direct search and global optimization algorithm developed by Storn and Price for continuous space optimization, which overcomes the drawbacks of conventional heuristic algorithms, i.e., Genetic Algorithm (GA) and Particle Swarm Optimization (PSO), etc. [23]. The advantages of ADE over the conventional heuristic algorithms include that (i) ADE can avoid being trapped in local optimum; (ii) ADE uses fewer tuning parameters. Only population size, maximum generation, differential weight $(F)$ bounds and crossover rate $(C R)$ bounds are adopted without resorting to an external probability density function; (iii) ADE exhibits better performance in exploration and exploitation by dynamically adjusting $F$ and CR [24-27]. In this paper, the ADE is adopted to optimize the objective function, which comprise the terms of distribution power loss and bus voltage deviations. Consequently, the proposed control can concurrently (i) regulate the bus voltages within the tolerances and (ii) reduce the distribution power loss on the distribution lines. This is possible, since the bus voltages of DC microgrids are generally allowed to have a certain degree of deviation, e.g., $5 \%$. The weighting factors of the two terms in the objective function are automatically tuned by the adaptive algorithm in [22], which further reduces the distribution power loss as compared to the weighting factors with fixed values. The proposed control consists of two layers. The secondary layer control is implemented by the ADE algorithm to provide bus voltage references for the primary layer control based on the measurements of bus voltages, line currents, and $\mathrm{SoC}$ of the batteries, while the primary layer control is achieved by local proportional-integral (PI) control to track the bus voltage references. SoC of the batteries are strictly controlled within the tolerances by including them in the constraints of the objective function. Verifications of the proposed control method are carried out in a 5-bus 48 V DC microgrid using a Real-Time Digital Simulator (RTDS). The proposed control method can also be used for larger-scale higher-voltage level DC microgrids. Compared to the conventional droop-based local control schemes, the cost saving from the distribution power loss mitigation by the proposed control scheme is more prominent than the additional installation and maintenance costs of the communication devices.

The major contribution of this paper is to mitigate the distribution power loss of DC microgrids by controlling the DBS, which has never been reported before. The novelty of the paper also includes 
the ADE-based control strategy with adaptive weighting factors, which is different from the traditional algorithms being exhibited in [20-22].

\section{Overview of the Investigated System and Control}

\subsection{Overview of a Standalone-DC Microgrid}

Figure 1 shows a typical architecture of a standalone DC microgrid, which comprises RES, DBS, and grid-connected converters, etc. The DBS are adopted to compensate the active power flow. When the supplied power by the RES is higher than the power demand by the loads, the DBS absorb the excessive power in the charging mode. Conversely, the DBS provide the deficient power in the discharging mode. However, the SoC of the battery packs in DBS are required to be controlled within the tolerances to prevent over-charge and deep-discharge. Thus, the spinning reserve activates when any one of the SoCs of the battery packs in DBS are higher than the upper limit. Conversely, the diesel generator activates when any one of the SoCs of the battery packs in DBS are lower than the lower limit.

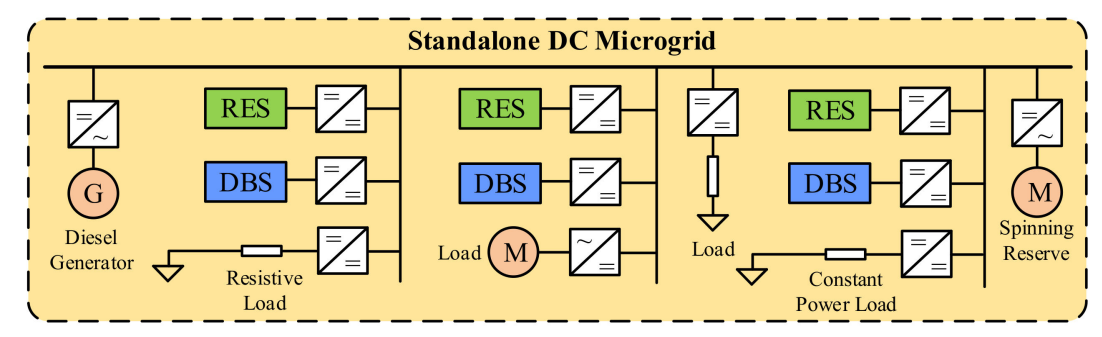

Figure 1. A typical architecture of standalone DC microgrid.

\subsection{Overview of a DBS with the Proposed Control Scheme in a Standalone-DC Microgrid}

The circuitry of a typical DBS in a standalone DC microgrid is depicted in Figure 2. A non-isolated boost converter is adopted as the grid-connected converter for the DBS. The proposed control scheme comprises two layers. The secondary layer is based on the measurements of all the bus voltages (i.e., $V_{\text {bus }}$ ), line currents (i.e., $I_{\text {line }}$ ) and SoC of the battery packs (i.e., $\mathrm{SoC}$ ) to provide bus voltage reference (i.e., $V_{\text {refi }}^{*}$ ) for the $i$-th DBS. The primary layer is based on the local measurements of the inductor current (i.e., $I_{\mathrm{b} i}$ ) and bus voltage (i.e., $V_{\text {busi }}$ ) of the $i$-th DBS.

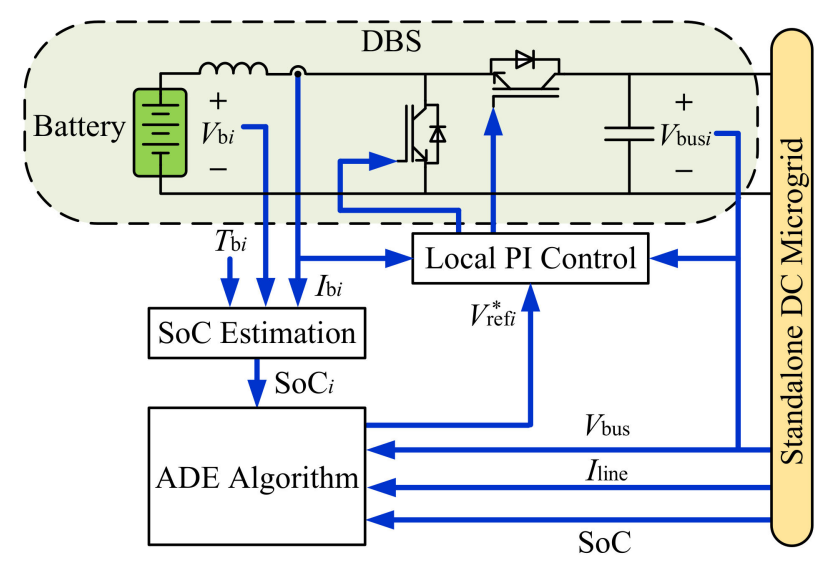

Figure 2. Circuitry of a typical distributed battery system (DBS) with the proposed control scheme in a standalone DC microgrid.

The flowchart of the proposed control scheme is shown in Figure 3. Initially, $V_{\text {bus }}, I_{\text {line }}$ and output voltage, current and temperature of the $i$-th battery pack (i.e., $V_{\mathrm{b} i}, I_{\mathrm{b} i}$ and $T_{\mathrm{b} i}$ ) are measured. The measured $V_{\mathrm{b} i}, I_{\mathrm{b} i}$ and $T_{\mathrm{b} i}$ are used to estimate the initial SoC of the battery pack (i.e., $\mathrm{SoC}_{i}(0)$ ) 
using a look-up table [28]. Then, the real-time SoC of the battery pack can be calculated by the Coulomb-counting method,

$$
\mathrm{SoC}_{i}=\operatorname{SoC}_{i}(0)-\frac{1}{C_{\mathrm{e} i}} \int I_{\mathrm{bi}} d t
$$

where $C_{\mathrm{e} i}$ is the capacity of the battery unit $i$. In practice, such a method of using both the look-up table and the Coulomb-counting method is widely adopted for industrial applications of batteries.

Once the SoC of the DBS are monitored, they can be used to be compared with upper and lower limits, i.e., $\mathrm{SoC}_{\max }$ and $\mathrm{SoC}_{\min }$, respectively. If any one of the monitored SoCs of the $\mathrm{DBS}$ is greater than the upper limit (i.e., $S_{0} C_{\max }$ ), the spinning reserves will operate to absorb redundant energy until all the SoCs of the DBS are within the tolerances. On the contrary, if any one of the monitored SoCs of the DBS is less than the lower limit (i.e., $\mathrm{So}_{\min }$ ), then the diesel generators will operate to provide sufficient energy until all the SoCs of the DBS are within the tolerances. The monitored SoC of the batteries in DBS and the measured $V_{\text {bus }}$ and $I_{\text {line }}$ are fed back to ADE algorithm, which generates $V^{*}$ refi for the local PI controllers. Since the grid-connected converter are boost converters, the classic cascaded current and voltage control strategy can be used based on $V^{*}$ refi and the feedback signals of $I_{\mathrm{b} i}$ and $V_{\mathrm{busi}}$, as shown in Figure 4 . Here, $K_{\mathrm{p} 1 i}$ and $K_{\mathrm{s} 1 i}$ are the tuning coefficients of the bus voltage PI controller. $I_{\text {bref } i}$ is the inductor current reference of the $i$-th DBS. $K_{\mathrm{p} 2 i}$ and $K_{\mathrm{s} 2 i}$ are the tuning coefficients of the inductor current PI controller. $d_{i}$ is the duty ratio of the boost converter of the $i$-th DBS. The time sequence of the proposed control scheme is plotted in Figure 5. Note that, the ADE optimization is conducted online. No additional offline training is required.

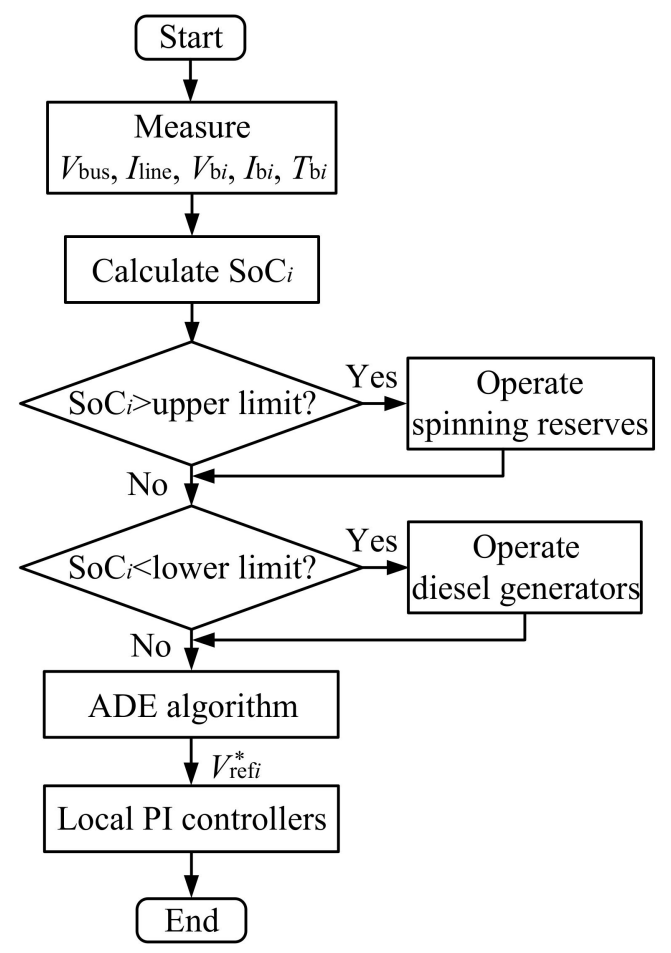

Figure 3. Flowchart of the proposed control scheme.

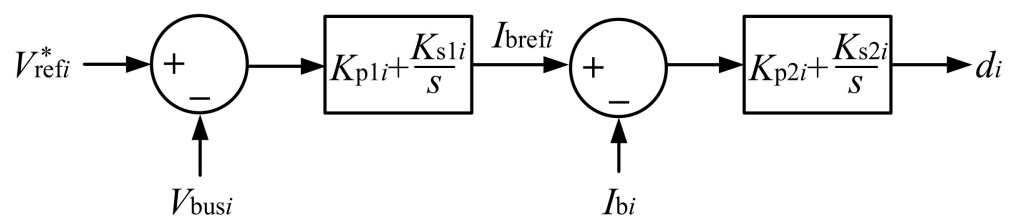

Figure 4. Control block diagram of the local proportional-integral (PI) control. 


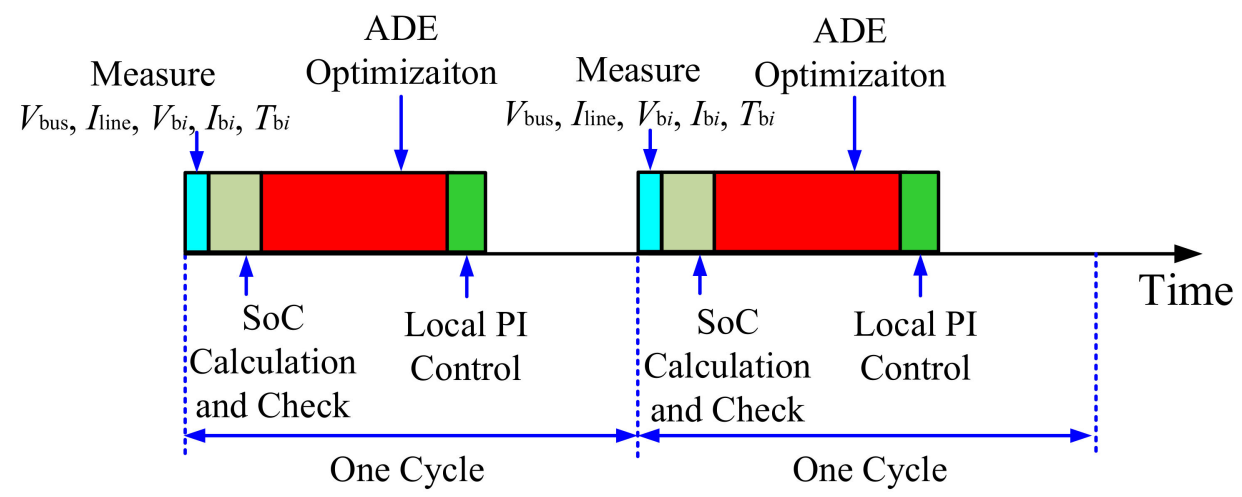

Figure 5. Time sequence of the proposed control scheme.

\section{Proposed ADE Algorithm}

Heuristic algorithms are widely adopted to solve the optimization problems with multiple objective constraints. However, the conventional heuristic algorithms, e.g., GA, run the risk of being trapped in local optimums. The unstable performances of the conventional heuristic algorithms are frequently observed with strong randomness. Beyond that, it is hard to choose parameters such as the number of population size, generations, crossover rate, and mutation rate to guarantee the path to be optimal. Instead, ADE can overcome these drawbacks of the conventional heuristic algorithms [24-27]. The proposed ADE algorithm consists of three stages: (i) initialization stage; (ii) iteration stage; (iii) final stage.

\section{[Initialization Stage]}

During this stage, the values of population size (i.e., $\left.P_{\text {size }}\right)$, generation maximum $\left(\max _{\text {gen }}\right)$ and stopping criteria in iterations are initialized. A random population of $P_{\text {size }}$ individuals (i.e., $V_{\text {bus }}$ ) is formed within the search-space bounds (i.e., [ $\left.\left.V_{\text {busmin }}, V_{\text {busmax }}\right]\right)$. Here, $V_{\text {busmin }}$ and $V_{\text {busmax }}$ are the lower bound and upper bound of bus voltage (Generally, $V_{\text {busmin }}=0.95 V_{\text {bus }}$ and $V_{\text {busmax }}=1.05 V_{\text {bus }}$ ).

\section{[Iteration Stage]}

For the conventional evolutionary algorithm (i.e., DE), the mutual operation performs at a low predefined probability (i.e., at 0.2 ) while the crossover operation is applied using a high value (i.e., 0.8 ). It is better to start with a high probability of mutation in the early generations and decrease it gradually. To achieve this, $\mathrm{ADE}$ adopts the adaptive mutual rate (i.e., $p_{\mathrm{m}}$ ) and adaptive crossover rate (i.e., $p_{\mathrm{c}}$ ), such that the algorithm is apt to more explorations of individual diversity in the early stage and is inclined to the exploitation afterwards.

The iteration stage of ADE comprises adaptive mutation, adaptive crossover, and selection operations. The main objective of mutation operation is to add some diversity by introducing more genetic materials into the population, to avoid the local optimum trap. For each individual $V_{\text {busi }}$ the mutation operation modifies $V_{\text {busi }}$ by a small differential variation to create a mutant $V_{\text {busm } i}$. As a result, the perturbation defines the direction and length of the search space. Specifically, three distinct individuals $V_{\text {busp1 }}, V_{\text {busp2 }}, V_{\text {busp3 }}$ are randomly selected with $\mathrm{p} 1 \neq \mathrm{p} 2 \neq \mathrm{p} 3$, and the difference vector, $V_{\text {busp2 }}-V_{\text {busp3 }}$, is calculated, and the mutant individual $V_{\text {busm } i}$ is then calculated as

$$
V_{\text {busm } i}=V_{\text {busp1 }}+p_{\text {mi } i}\left(V_{\text {busp2 }}-V_{\text {busp3 }}\right)
$$

where $p_{\mathrm{m} i}\left(V_{\mathrm{busp} 2}-V_{\mathrm{busp} 3}\right)$ represents the mutation step size. All the mutants $V_{\mathrm{busm} i}$ are recorded in a mutant vector $\mathbf{V}_{\text {busm }} \cdot p_{\mathrm{m} i}$ is the mutation rate used to control the amplification of the differential variation. Compared to the conventional Differential Evolution (DE) of using a fixed value of $p_{\mathrm{m}}$, the mutation rate of the ADE is adaptively generated for each individual as

$$
p_{\mathrm{m} i}=F_{\min }+\left(F_{\max }-F_{\min }\right) \frac{f_{2}-f_{1}}{f_{3}-f_{1}}
$$


where $F_{\min }$ and $F_{\max }$ are the minimum and maximum mutation rate. $f_{1}, f_{2}$ and $f_{3}\left(f_{1}<f_{2}<f_{3}\right)$ are the fitness values of $V_{\text {busp1 }}(g), V_{\text {busp2 }}(g), V_{\text {busp3 }}(g)$ based on the objective function

$$
\begin{gathered}
\min J=\alpha \sum_{r=1}^{m}\left|V_{\text {rate }}-V_{\text {busi }}\right|+(1-\alpha) \sum_{i, j=1}^{m} I_{\text {line } i j}\left|V_{\text {busi }}-V_{\text {bus } j}\right| \\
\text { s.t. } 0 \leq \alpha \leq 1 \\
V_{\text {busmin }} \leq V_{\text {busi }} \leq V_{\text {busmax }} \\
\operatorname{SoC}_{\min } \leq \mathrm{SoC}_{i} \leq \mathrm{SoC}_{\max }
\end{gathered}
$$

where $V_{\text {rate }}$ is the rated DC bus voltage. $I_{\text {lineij }}$ is the line current between the bus $i$ and the bus $j$. $V_{\text {busi }}$ and $V_{\text {busj }}$ are the DC bus voltages of the bus $i$ and the bus j. $\alpha$ is the weighting factor, which is tuned by the adaptive algorithm in [22]. The flowchart of the adaptive weighting factor tuning algorithm is depicted in Figure 6. The weighting factor is initialized and adopted to the objective function first. Then, all the bus voltages are checked. If all the bus voltages are within the tolerances, the weighting factor is reduced by $\Delta \alpha$ (i.e., $\alpha(\kappa+1)=\alpha(\kappa)-\Delta \alpha$ ) in case of $\alpha(\kappa)-\Delta \alpha \geq 0$ or keeps invariant (i.e., $\alpha(\kappa+1)=\alpha(\kappa)$ ) in case of $\alpha(\kappa)-\Delta \alpha<0$ at the next sampling time $\kappa+1$. This process reiterates until one of the bus voltages is beyond the tolerances. If so, the weighting factor is increased by $\Delta \alpha$ (i.e., $\alpha(\kappa+1)=\alpha(\kappa)+\Delta \alpha$ ) in case of $\alpha(\kappa)+\Delta \alpha \leq 1$ or keeps invariant (i.e., $\alpha(\kappa+1)=\alpha(\kappa)$ ) in case of $\alpha(\kappa)-\Delta \alpha>1$ at the next sampling time $\kappa+1$. This process reiterates until all the bus voltages are within the tolerances. The adaptive weighting factor tuning algorithm guarantees that the power loss on the distribution lines can be approximately optimized at different power generation and load conditions by searching out the optimal $\alpha$ for the objective function.

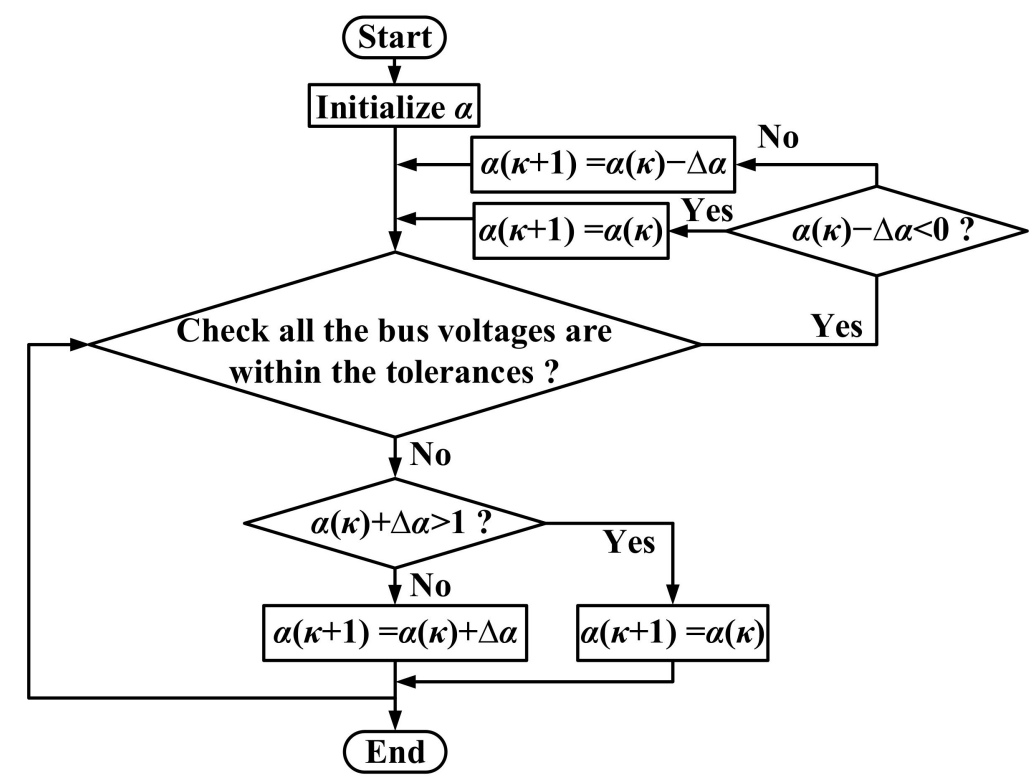

Figure 6. Flowchart of the adaptive weighting factor tuning algorithm.

Then, the crossover operator amalgamates the mutant vector $\mathbf{V}_{\text {busm }}$ and the parent vector $\mathbf{V}_{\text {bus }}$ to create a trial vector $\mathbf{V}_{\text {bust }}$. Generally, the crossover operation can be implemented in either binomial or exponential. Due to that the binomial being more straightforward and more frequently used, it is adopted for the crossover operation in this paper as

$$
V_{\text {bust } i}=\left\{\begin{array}{l}
V_{\text {busmi }}, \quad r<p_{c i} \\
V_{\text {busi }} \text { others }
\end{array}\right.
$$


where $r \sim N(0,1)$ is a normal distribution. All the trial individuals $V_{\text {busti }}$ are recorded in a trial vector $\mathbf{V}_{\text {bust }}$. Each offspring is a stochastic linear combination of three randomly selected individuals when $r<p_{\mathrm{c} i}$. Otherwise, the offspring inherits from the parent vector. Superior to the conventional DE of using a fixed value of $p_{\mathrm{c}}$, the crossover rate of the ADE is adaptively generated for each individual as

$$
p_{\mathrm{c} i}=\left\{\begin{array}{cc}
p_{\min }+\frac{\left(p_{\max }-p_{\min }\right)\left(f_{i}-f_{\min }\right)}{f_{\min }-f_{\min }}, & f_{i}<f \\
p_{\min }, & f_{i} \geq f
\end{array}\right.
$$

where $p_{\min }$ and $p_{\max }$ are the minimum and maximum crossover rate. $f_{\min }, f_{\max }$ and $f$ are the minimum, maximum and average objectives of $V_{\text {busp } 1}(g), V_{\text {busp2 }}(g), V_{\text {busp } 3}(g) . f_{i}$ is the fitness values of the current individual. Since the crossover rate $p_{\mathrm{c} i}$ is adaptive, it makes better offspring that are more likely to survive until the next generation, as compared to the fixed crossover rate. The successful crossover rate of the last generation would be used to guide the generation of new offspring.

The third operation of ADE in the iteration stage is selection, which is similar to the elitism operation of the conventional heuristic algorithms. Selection operation is conducted based on one-to-one competition between the individuals in the parent vector and the trial vector at every generation. Better individuals are chosen for the next generation. Specifically, if the fitness value of the objective function based on the trial vector is less than or equal to that of the parent vector $\mathbf{V}_{\text {bus }}$, the trial vector will survive and be used for the next generation. Otherwise, the parent vector will be adopted for the next generation.

\section{[Final Stage]}

Two terminal criteria of ADE include the maximum generation max $_{\text {gen }}$ and the maximum deviation rate $\max _{\mathrm{dr}}$. In the final stage, the algorithm stops when any one of the terminal criteria is satisfied. Pseudocode of the whole ADE Algorithm (Algorithm 1) is provided as follows:

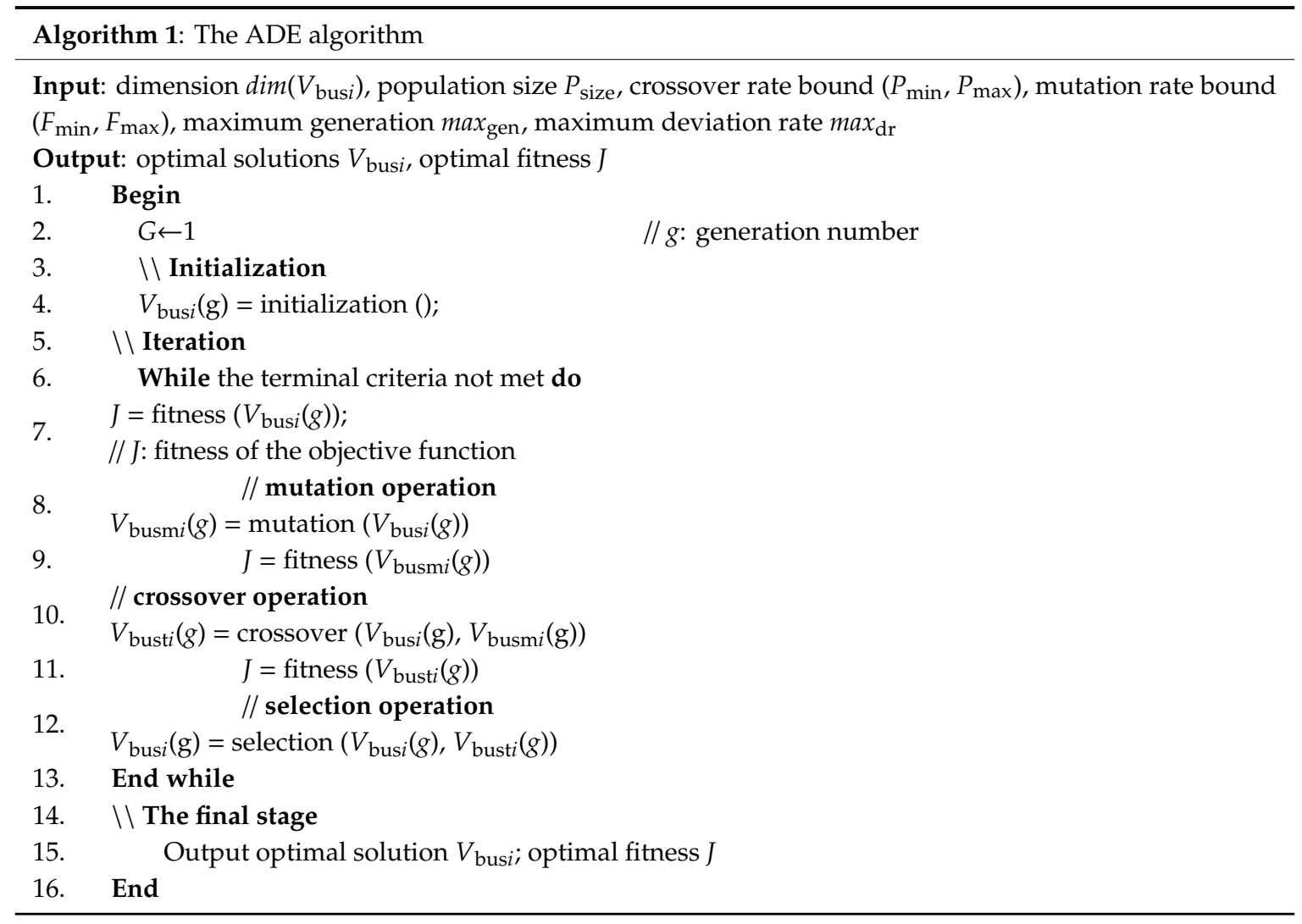




\section{Case Studies}

To verify the performance of the proposed ADE-based control strategy, A 5-bus $48 \mathrm{~V}$ DC microgrid is built in RSCAD and tested using the RTDS with a sampling frequency of $1 \mathrm{kHz}$. RSCAD is the proprietary simulation software package that is used to configure the simulations that are then run on parallel processing hardware in RTDS. In this paper, the measured voltages and currents in RSCAD are exported to the Matlab, in which the ADE algorithm calculates the optimal bus voltages. Then, the calculated optimal bus voltages are exported from Matlab to RSCAD for local bus voltage regulations. The topology of the 5-bus DC microgrid is shown in Figure 7. The sampling frequency and switching frequency of the grid-connected boost converter $20 \mathrm{kHz}$ and $10 \mathrm{kHz}$, respectively. The communication delay of the signals from the measurements to the ADE algorithm is uniformly set to be $0.5 \mathrm{~s}$. The capacity of the battery packs (i.e., $C_{\mathrm{e} i}$ ) are identical for all the DBS, which is $100 \mathrm{Ah} . \mathrm{SoC}_{\min }$ and $\mathrm{SoC}_{\max }$ for all the DBS are $20 \%$ and $90 \%$, respectively. The initial weighting factor (i.e., $\alpha(0)$ ) is 1 . The weighting factor difference (i.e., $\Delta \alpha$ ) for the adaptive weighting factor algorithm is 0.1 , which can guarantee both significant power loss and fast computation speed.

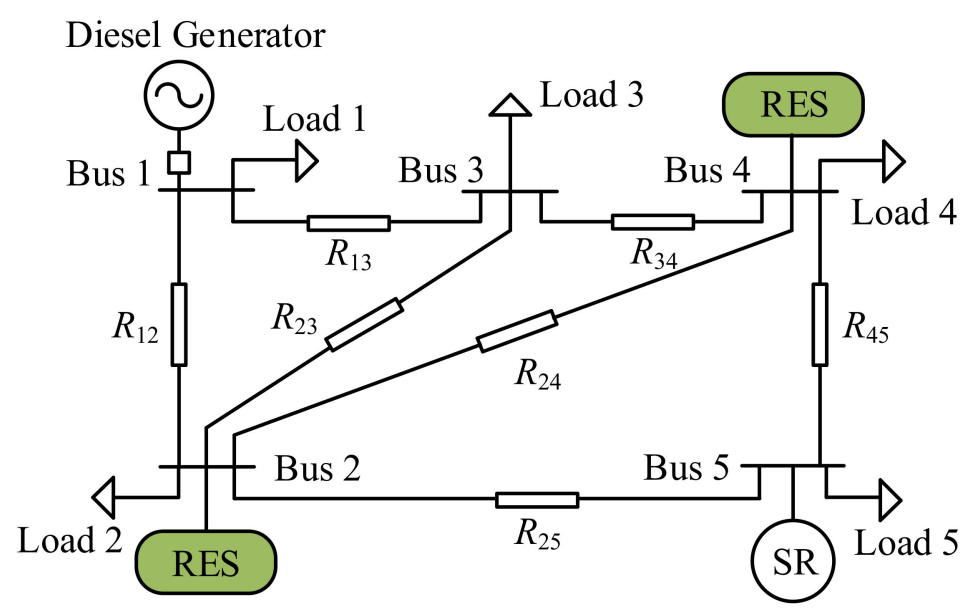

Figure 7. Topology of the 5-bus 48 V DC microgrid.

In the 5-bus DC microgrid, two RES are installed at the at buses 2 and 4, respectively. The diesel generator is installed at the bus 1 . Spinning reserves are installed at bus 5 . All the buses are connected with loads. The specifications of the 5-bus DC microgrid and the parameters of the adopted controllers are listed in Tables 1 and 2, respectively. Here, the tuning parameters of the local PI controllers (i.e., $K_{\mathrm{p} 1}, K_{\mathrm{i} 1}, K_{\mathrm{p} 2}$ and $K_{\mathrm{i} 2}$ ) are identical for all the DBS.

Table 1. Specifications of the 5-bus DC microgrid.

\begin{tabular}{cccc}
\hline Parameter & Value & Parameter & Value \\
\hline$R_{12}$ & $0.2 \Omega$ & $R_{13}$ & $0.2 \Omega$ \\
$R_{23}$ & $0.3 \Omega$ & $R_{24}$ & $0.45 \Omega$ \\
$R_{25}$ & $0.4 \Omega$ & $R_{34}$ & $0.2 \Omega$ \\
$R_{45}$ & $0.2 \Omega$ & Load 1 & $5 \Omega$ \\
Load 2 & $10 \Omega$ & Load 3 & $1.5 \mathrm{~kW}$ \\
Load 4 & $0.5 \mathrm{~kW}$ & Load 5 & $1 \mathrm{~kW}$ \\
$V_{\text {busmin }}$ & $45.6 \mathrm{~V}$ & $V_{\text {busmax }}$ & $50.4 \mathrm{~V}$ \\
\hline
\end{tabular}


Table 2. Parameters of the adopted control.

\begin{tabular}{cc}
\hline Parameter & Value \\
\hline Population size $P_{\text {size }}$ & 18 \\
Maximum generation max $_{\text {gen }}$ & 400 \\
Lower limit of the mutation rate $F_{\min }$ & 0.4 \\
Upper limit of the mutation rate $F_{\max }$ & 0.8 \\
Lower limit of the crossover rate $P_{\min }$ & 0.1 \\
Upper limit of the crossover rate $P_{\max }$ & 0.6 \\
$K_{\mathrm{p} 1}$ & 0.2 \\
$K_{\mathrm{i} 1}$ & 0.1 \\
$K_{\mathrm{p} 2}$ & 0.1 \\
$K_{\mathrm{i} 2}$ & 0.1 \\
\hline
\end{tabular}

When the power of RES at buses 2 and 4 (i.e., $P_{\mathrm{g} 1}$ and $P_{\mathrm{g} 2}$ ) fluctuate in $12 \mathrm{~h}$, as shown in Figure $8 \mathrm{a}$, the bus voltages will fluctuate if no DBS is installed. The corresponding bus voltages are shown in Figure 8b. Apparently, the bus voltages will exceed the tolerances.

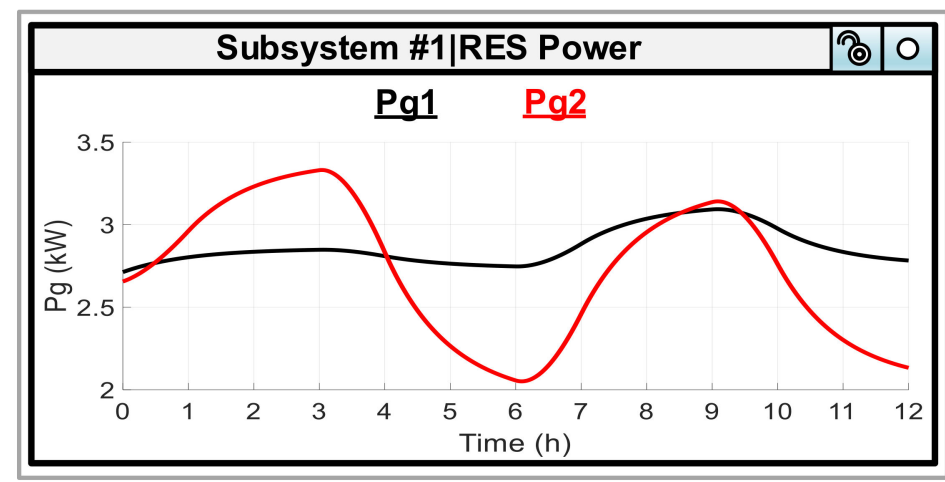

(a) RES power

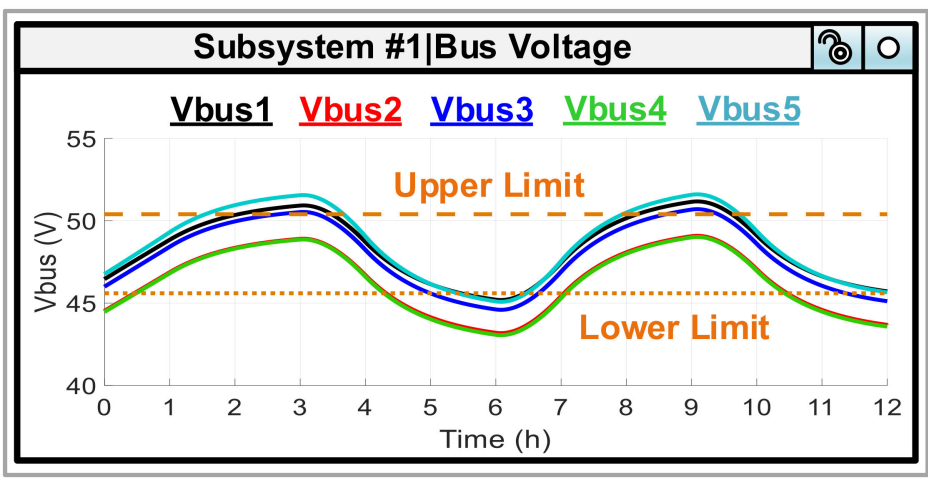

(b) bus voltages

Figure 8. Renewable energy sources (RES) power and bus voltages of the 5-bus DC microgrid without DBS.

However, when DBS are installed at the buses 2, 4 and 5 with the initial SoC of $50 \%, 40 \%$ and $60 \%$, respectively. The DBS are only controlled by the local PI controllers to track the rated bus voltage, while the ADE algorithm is not activated. Here, the local PI controllers are designed based on the control method in Figure 4 by incorporating an SoC outer loop control. Details of the local PI controllers being adopted here can be found in [9]. Besides, the flowchart of the control algorithm also includes the 
check of SoC whether beyond the tolerances or not. If the SoC of the battery packs are greater than the upper limit, the spinning reserve operates. Conversely, if the SoC of the battery packs are less than the lower limit, the diesel generator activates. The waveforms of the bus voltages are shown in Figure 9. Apparently, the fluctuations of the bus voltages are mitigated by the local PI controllers.

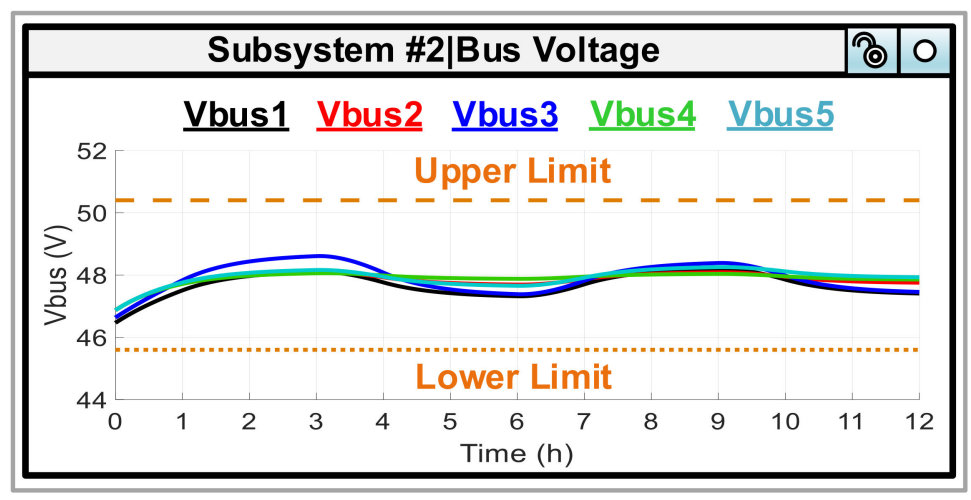

Figure 9. Bus voltages of the 5-bus DC microgrid (with only RES fluctuations) with DBS only controlled by the local PI controllers.

Then, the ADE algorithm with adaptive weighting factor is executed (the initial SoC of the DBS at the buses 2, 4 and 5 are $50 \%, 40 \%$ and $60 \%$, respectively). Figure 10 shows the resulting bus voltages in $12 \mathrm{~h}$. Obviously, all the bus voltages are still regulated within the tolerances. The corresponding fitness value, adaptive weighting factor and SoC of the battery packs are plotted in Figures 11 and 12, respectively. SoC2, SoC4 and SoC5 indicate the SoC of the battery packs at buses 2, 4 and 5 . In Figure 12, all the SoC of the battery packs are controlled within the boundaries, i.e., $\mathrm{SoC}_{\mathrm{min}}=$ $20 \%$ and $\mathrm{SoC}_{\max }=90 \%$.

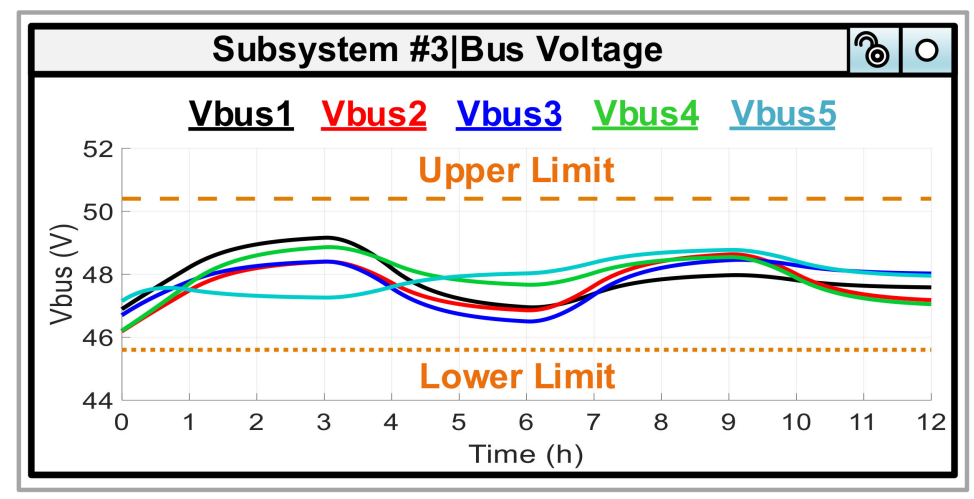

Figure 10. Bus voltages of the 5-bus DC microgrid (with only RES fluctuations) with DBS controlled by the proposed control.

The comparative waveforms of the distribution power loss of the 5-bus DC microgrid between the DBS with only local PI control (i.e., Ploss1) and with the proposed control (i.e., Ploss2) are shown in Figure 13. Apparently, the distribution power loss are reduced by the proposed control. By integrating the distribution power loss over the 12-h period, the distribution energy loss can be calculated. The distribution energy loss for the DBS with only local PI control and the proposed control are $3.35 \mathrm{kWh}$ and $2.35 \mathrm{kWh}$, respectively. The energy saving is about $29.85 \%$ by using the proposed control in lieu of DBS with only local PI control. 


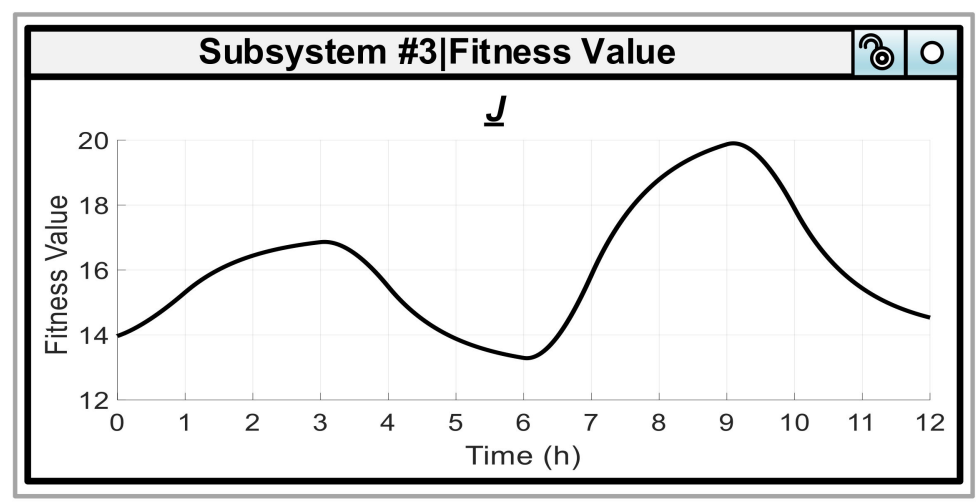

(a) fitness value

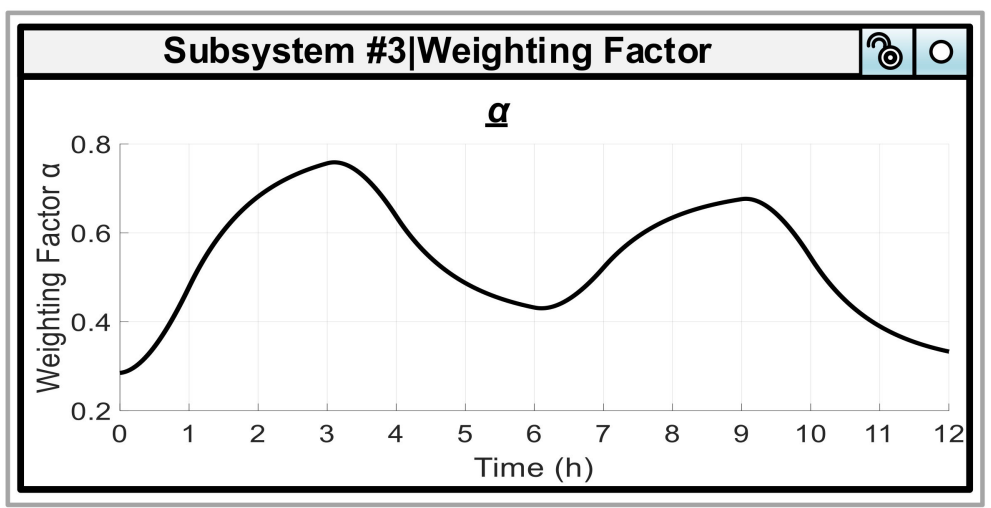

(b) adaptive weighting factor

Figure 11. Fitness value and adaptive weighting factor of the proposed control for the 5-bus DC microgrid with only RES fluctuations.

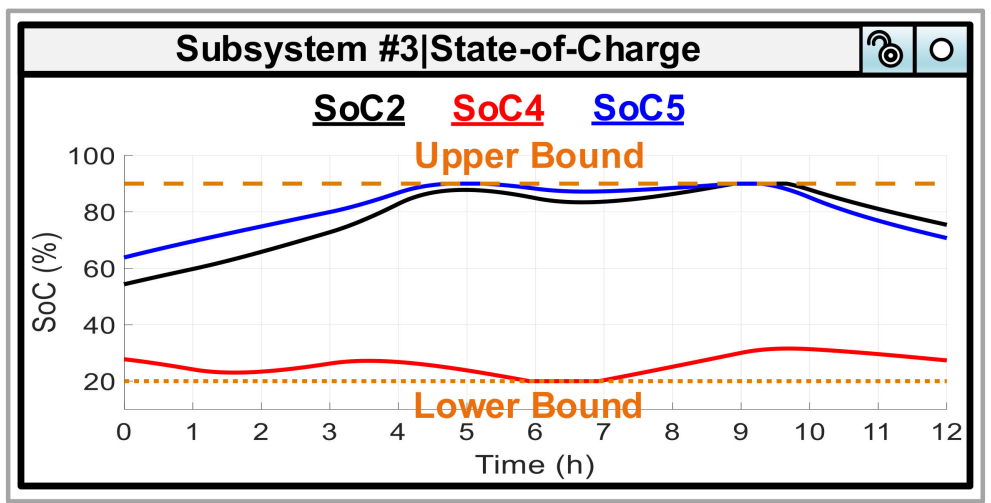

Figure 12. State-of-charge $(\mathrm{SoC})$ of the battery packs in the 5-bus DC microgrid with only RES fluctuations.

The proposed ADE algorithm is also adopted to reduce the distribution power loss of the 5-bus DC microgrid with both RES variations and load changes. The fluctuations of RES power at buses 2 and 4 are shown in Figure 8a, while load 3, load 5, and load 2 are changed from $1.5 \mathrm{~kW}$ to $3 \mathrm{~kW}, 1 \mathrm{~kW}$ to $0.5 \mathrm{~kW}$, and $10 \Omega$ to $20 \Omega$ at the $3 \mathrm{rd}-$, 6th-, and 9th-hour, respectively. The initial SoC of the DBS at buses 2,4 and 5 are $50 \%, 40 \%$ and $60 \%$, respectively. Figure 14 shows the corresponding bus voltages in $12 \mathrm{~h}$. Obviously, all the bus voltages are regulated within the tolerances. 


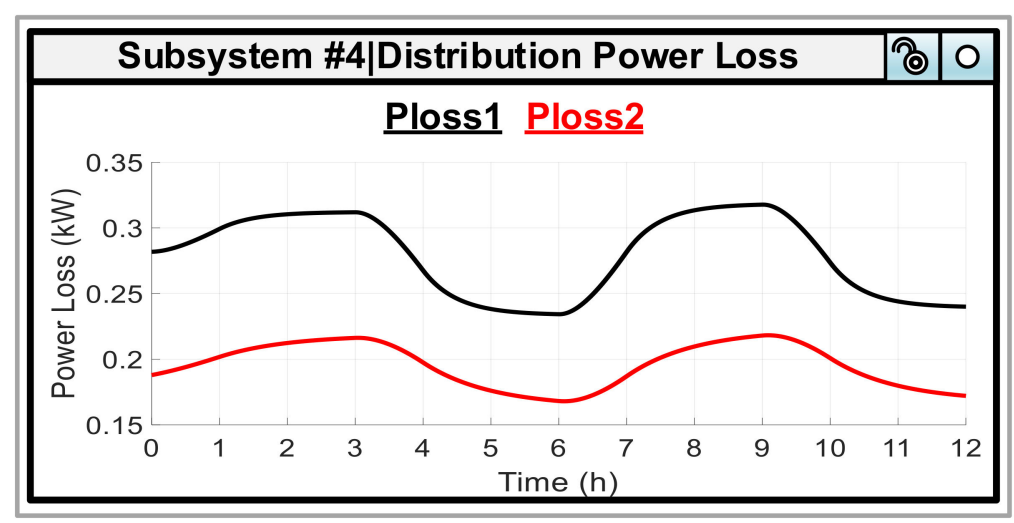

Figure 13. Distribution power loss of the 5-bus DC microgrid (with only RES fluctuations) with DBS controlled by two control schemes.

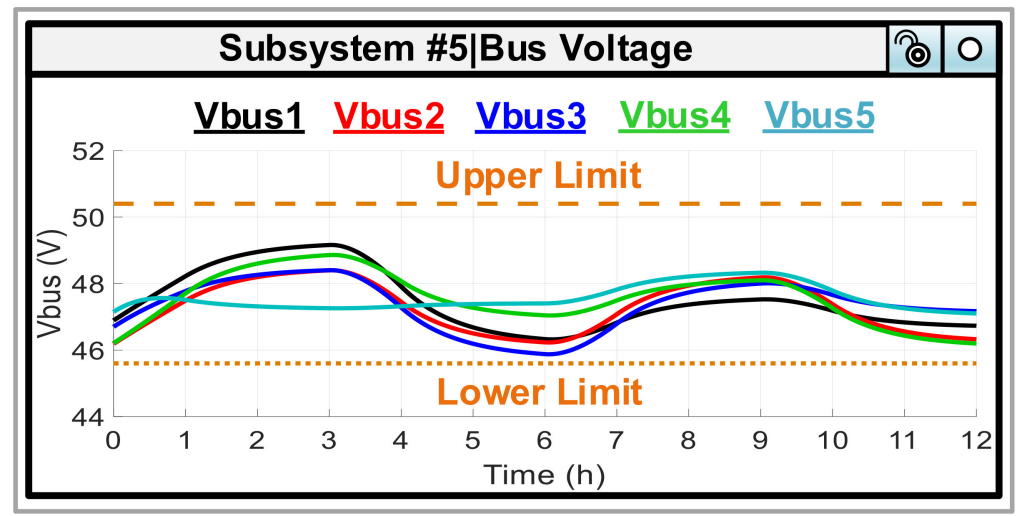

Figure 14. Bus voltages of the 5-bus DC microgrid (with both RES and load variations) with DBS controlled by the proposed control.

The corresponding SoC of the battery packs are shown in Figure 15. Apparently, all the SoC of the battery packs are regulated within the tolerances, i.e., $\mathrm{SoC}_{\min }=20 \%$ and $\mathrm{SoC}_{\max }=90 \%$. The comparative waveforms of the distribution power loss between the conventional local PI control and the proposed control are depicted in Figure 16. Obviously, the distribution power loss is mitigated for the 5-bus DC microgrid with both RES and load variations. By integrating the distribution power loss over the 12-h period, the distribution energy loss for both control methods are $3.82 \mathrm{kWh}$ and $2.41 \mathrm{kWh}$, respectively. The energy saving is about $36.91 \%$.

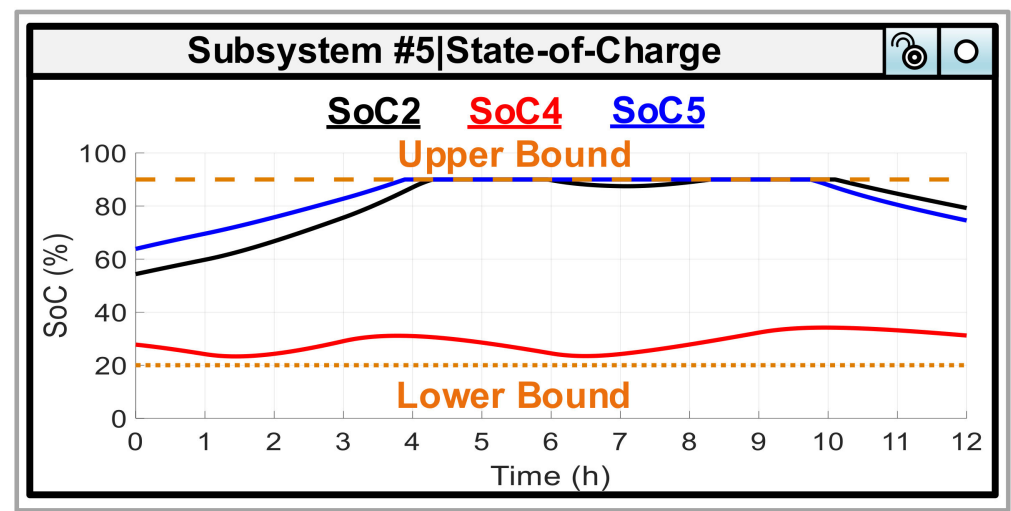

Figure 15. SoC of the battery packs in the 5-bus DC microgrid with both RES and load variations. 


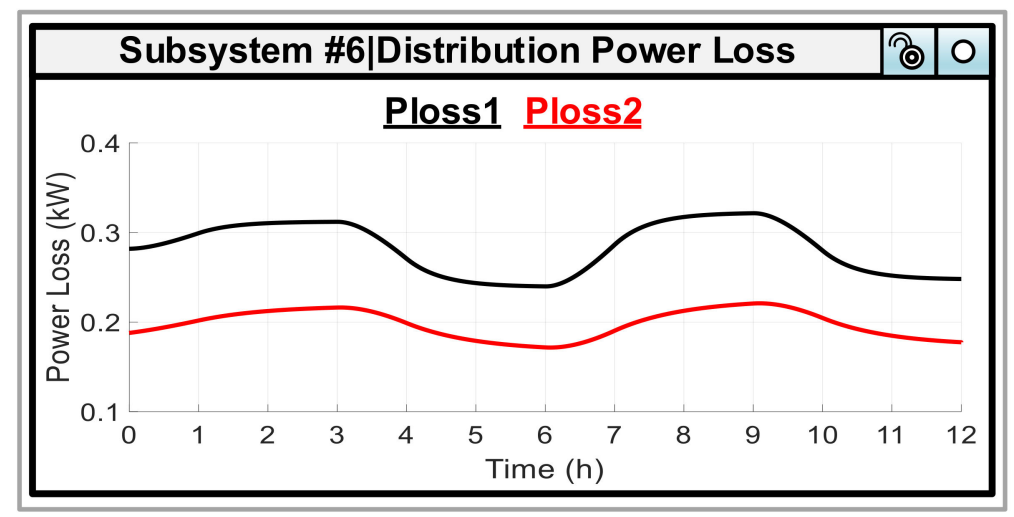

Figure 16. Distribution power loss of the 5-bus DC microgrid (with both RES and load variations) with DBS controlled by two control schemes.

From the case study on the 5-bus $48 \mathrm{~V} \mathrm{DC}$ microgrid, the proposed ADE-based control scheme is validated, showing that it reduces the distribution power loss on the distribution lines of DC microgrids while still regulating the bus voltages within the tolerances. The SoC of the battery packs in DBS are also stringently controlled within the boundaries, such that the lifespan of the battery packs can be prolonged over long-time operations. The average time consumed by the ADE algorithm is only about $10 \mathrm{~s}$. The period time consumed by the proposed control method, which includes the measurement time of voltages and currents, calculation time of SoC, computation time of the ADE algorithm, and the operating time of local PI controllers, is negligible as compared to the variation time of the renewable energy power.

\section{Conclusions}

Unlike the conventional droop control to only regulate the bus voltages and state-of-charge (SoC) of the battery packs in standalone DC microgrids, an Adaptive Differential Evolution (ADE)-based control scheme is proposed in this paper to further mitigate the power loss on the distribution lines online. This might be the first paper to report the mitigation of the distribution power loss as well as bus voltage regulations by controlling DBS in DC microgrid. Being different from the existing algorithms to reduce the distribution power loss in DC microgrids, the adopted control strategy uses the ADE algorithm with adaptive weighting factors, and therefore the numbers of tuning parameters are decreased. Case studies using a Real-Time Digital Simulator (RTDS) reveal that the distribution energy loss can save about $29.85 \%$ and $36.91 \%$ for 5 -bus $48 \mathrm{~V} \mathrm{DC}$ microgrids with only RES fluctuations and with both RES and load variations by the proposed control, as compared to the conventional local proportional-integral (PI) control, while the bus voltages and the SoC of the battery packs are still regulated within the tolerances.

Author Contributions: Conceptualization, Y.M. and Y.Y.; methodology, Y.M.; software, J.D.; validation, J.D. and Y.M.; formal analysis, J.D.; investigation, J.D.; resources, J.D.; data curation, J.D.; writing—original draft preparation, J.D.; writing - review and editing, Y.Y.; visualization, J.D.; supervision, Y.Y. All authors have read and agreed to the published version of the manuscript.

Funding: This research received no external funding.

Acknowledgments: The authors would like to thank the anonymous reviewers.

Conflicts of Interest: The authors declare no conflict of interest.

\section{References}

1. Gragicevic, T.; Lu, X.; Vasquez, J.; Guerrero, J. DC microgrid-part II: A review of power architectures, applications, and standardization issues. IEEE Trans. Power Electron. 2016, 31, 3528-3549. [CrossRef] 
2. Peyghami, S.; Dvari, P.; Mokhari, H.; Blaabjerg, F. Decentralized droop control in DC microgrids based on frequency injection. IEEE Trans. Smart Grid 2019, 10, 6782-6791. [CrossRef]

3. Zhang, Y.; Li, Y.W. Energy management strategy for supercapacitor in droop-controlled DC microgrid using virtual impedance. IEEE Trans. Power Electron. 2017, 32, 2704-2716. [CrossRef]

4. Boroyevich, D.; Cvetkovic, I.; Dong, D.; Burgos, R.; Wang, F.; Lee, F. Future electronic power distribution systems a contemplative view. In Proceedings of the International Conference on Optimization of Electrical and Electronic Equipment (OEEE), Basov, Romania, 15 July 2010.

5. Guerrero, J.M.; Vasquez, J.C.; Matas, J.; Vicuna, L.G.; Castilla, M. Hierarchical control of droop-controlled AC and DC microgrids-a general approach toward standardization. IEEE Trans. Ind. Electron. 2011, 58, 158-172. [CrossRef]

6. Augustine, S.; Mishra, M.K.; Lakshminarasmma, N. Adaptive droop control strategy for load sharing and circulating current minimization in low-voltage standalone DC microgrid. IEEE Trans. Sustain. Energy 2015, 6, 132-141. [CrossRef]

7. Dizqah, A.M.; Maheri, A.; Busawon, K.; Kamjoo, A. A multivariable optimal energy management strategy for standalone DC microgrids. IEEE Trans. Power Syst. 2015, 30, 2278-2287. [CrossRef]

8. Yang, Y.; Mok, K.T.; Tan, S.C.; Hui, S.Y.R. Nonlinear dynamic power tracking of low-power wind energy conversion system. IEEE Trans. Power Electron. 2015, 30, 5223-5236. [CrossRef]

9. Yang, Y.; Qin, Y.; Tan, S.C.; Hui, S.Y.R. Efficiency improvement of photovoltaic-battery systems in standalone DC microgrids using a local hierarchical control for the battery system. IEEE Trans. Power Electron. 2019, 34, 10796-10807. [CrossRef]

10. Zhou, H.; Bhattacharya, T.; Tran, D.; Siew, T.; Khambadkone, A. Composite energy storage system involving battery and ultracapacitor with dynamic energy management in microgrid applications. IEEE Trans. Power Electron. 2011, 26, 923-930. [CrossRef]

11. Gao, F.; Bozhko, S.; Asher, G.; Wheeler, P.; Patel, C. An improved voltage compensation approach in a droop-controlled DC power system for the more electric aircraft. IEEE Trans. Power Electron. 2016, 31, 7369-7383.

12. Prabhakaran, P.; Goyal, Y.; Agarwal, V. Novel nonlinear droop control techniques to overcome the load sharing and voltage regulation issues in DC microgrids. IEEE Trans. Power Electron. 2018, 33, 4477-4487. [CrossRef]

13. Li, X.; Guo, L.; Zhang, S.; Wang, C.; Li, Y.W.; Chen, A.; Feng, Y. Observer-based DC voltage droop and current feed-forward control of a DC microgrid. IEEE Trans. Smart Grid 2018, 9, 5207-5216. [CrossRef]

14. Liu, C.; Zhao, J.; Wang, S.; Lu, W.; Qu, K. Active identification method for line resistance in DC microgrid based on single pulse injection. IEEE Trans. Power Electron. 2018, 33, 5561-5564. [CrossRef]

15. Jiang, Y.; Yang, Y.; Tan, S.C.; Hui, S.Y.R. Adaptive current sharing of distributed battery systems in DC microgrids using adaptive virtual resistance-based droop control. In Proceedings of the IEEE Energy Conversion Congress and Exposition (ECCE), Baltimore, MD, USA, 29 September-3 October 2019.

16. Lu, X.; Sun, K.; Guerrero, J.M.; Vasquez, J.C.; Huang, L. Double-quadrant state-of-charge-based droop control method for distributed energy storage systems in autonomous DC microgrids. IEEE Trans. Smart Grid 2015, 6, 147-157. [CrossRef]

17. Hamad, A.A.; Azzouz, M.A.; El-Saadany, E.F. Multiagent supervisory control for power management in DC microgrids. IEEE Trans. Smart Grid 2016, 7, 1057-1068. [CrossRef]

18. Dufo-Lopez, R.A.; Bernal-Agustin, J.L.; Yusta-Loyo, J.M.; Dominguez-Navarro, J.A.; Ramirez-Rosado, I.J.; Lujano, J.; Aso, I. Multi-objective optimization minimizing cost and life cycle emissions of stand-alone PV-wind-diesel systems with batteries storage. Appl. Energy 2011, 88, 4033-4041. [CrossRef]

19. Qian, X.; Yang, Y.; Li, C.; Tan, S.C. Economic dispatch of DC microgrids under real-time pricing using adaptive differential evolution algorithm. In Proceedings of the IEEE Power Electronics and Motion Control Conference (IPEMC 2020-ECCE Asia), Nanjing, China, 31 May-3 June 2020.

20. Yang, Y.; Tan, S.C.; Hui, S.Y.R. Mitigating distribution power loss of DC microgrids with DC electric springs. IEEE Trans. Smart Grid 2018, 9, 5897-5906. [CrossRef]

21. Jiang, Y.; Yang, Y.; Tan, S.C.; Hui, S.Y.R. Mitigating distribution power losses of standalone AC microgrids using particle-swarm-optimization control for distributed battery systems. In Proceedings of the IEEE Applied Power Electronics Conference and Exposition (APEC), Anaheim, CA, USA, 17-21 March 2019. 
22. Yang, Y.; Qin, Y.; Tan, S.C.; Hui, S.Y.R. Reducing distribution power loss of islanded AC microgrids using distributed electric springs with predictive control. IEEE Trans. Ind. Electron. 2020, 99, 1. [CrossRef]

23. Storn, R.; Price, K. Differential evolution-a simple and efficient heuristic for global optimization over continuous spaces. J. Glob. Optim. 1997, 11, 341-359. [CrossRef]

24. Tusar, T.; Filipic, B. Differential evolution versus genetic algorithms in multiobjective optimization. Evol. Multi-Criterion Optim. 2007, 1, 257-271.

25. Yang, Y.; Tan, S.C.; Hui, S.Y.R. Front-end parameter monitoring method based on two-layer adaptive evolution for SS-compensated wireless power transfer system. IEEE Trans. Ind. Informat. 2019, 15, 6101-6113. [CrossRef]

26. Mao, Y.; Niu, S.; Yang, Y. Differential evolution-based multiobjective optimization of the electrical continuously variable transmission system. IEEE Trans. Ind. Electron. 2018, 65, 2080-2089. [CrossRef]

27. Brest, J.; Zumer, V.; Maucec, M.S. Self-adaptive differential evolution algorithm in constrained real-parameter optimization. In Proceedings of the IEEE International Conference on Evolutionary Computation, Vancouver, BC, Canada, 16-21 July 2006.

28. Zhang, R.; Xia, B.; Li, B.; Cao, L.; Lai, Y.; Zheng, W.; Wang, H.; Wang, W. State of the art of lithium-ion battery SOC estimation for electric vehicles. Energies 2018, 11, 1820. [CrossRef]

(C) 2020 by the authors. Licensee MDPI, Basel, Switzerland. This article is an open access article distributed under the terms and conditions of the Creative Commons Attribution (CC BY) license (http://creativecommons.org/licenses/by/4.0/). 